\title{
Tricuspid Valve Disease, CTCAE
}

National Cancer Institute

\section{Source}

National Cancer Institute. Tricuspid Valve Disease, CT CAE. NCI Thesaurus. Code C143889.

A disorder characterized by a defect in tricuspid valve function or structure. 